\title{
A new hybrid explicit/implicit in-plane-out-of-plane separated representation for the solution of dynamic problems defined in plate-like domains
}

\author{
G. Quaranta ${ }^{\text {a }}$, B. Bognet ${ }^{a}$, R. Ibañez ${ }^{\text {a }}$, A. Tramecon ${ }^{\text {b }}$, E. Haug ${ }^{\text {b }}$, F. Chinesta ${ }^{\text {c,* }}$ \\ ${ }^{a}$ ESI GROUP Chair E High Performance Computing Institute, Ecole Centrale Nantes, 1 rue de la Noe, BP 92101, F-44321 Nantes cedex 3, France \\ ${ }^{\mathrm{b}}$ ESI GROUP, 99 rue des Solets, 94513 Rungis, France \\ ${ }^{\mathrm{c}}$ ESI GROUP Chair E PIMM Laboratory, ENSAM ParisTech, 151 Boulevard de l'Hôpital, F-75013 Paris, France
}

\section{A R T I C L E I N F O}

Article history:

Received 28 October 2017

Accepted 1 May 2018

Available online 8 September 2018

\section{Keywords:}

Dynamics

Implicit

Explicit

Hybrid time discretization

Plates

Laminates

PGD

Separated representation

\begin{abstract}
A B S T R A C T
The present paper extends in-plane-out-of-plane separated representations successfully used for addressing fully 3D model solutions defined in plate-like domain, to dynamics. Common time integration are performed using explicit or implicit strategies. Even if the implementation of implicit integration schemes into a 3D in-plane-out-of-plane separated representation does not imply major difficulties, the use of explicit integration preferable in many applications becomes a tricky issue. In fact the mesh employed for discretizing the out-of-plane dimension (thickness) determines the maximum time-step ensuring stability. In this paper we introduce a new efficient hybrid explicit/implicit in-plane-out-ofplane separated representation for dynamic problems defined in plate-like domains that allows computing 3D solutions with the stability constraint exclusively determined by the coarser in-plane discretization.
\end{abstract}

\section{Introduction}

Many mechanical systems and complex structures involve plate and shell parts whose main particularity is having a characteristic dimension (the one related to the thickness) much lower that the other ones (in-plane dimensions). The introduction of appropriate kinematic and mechanic hypotheses allow the reduction of the general 3D mechanical problem to a $2 \mathrm{D}$ involving the in-plane coordinates. This was the route employed for deriving beam, plate and shell theories in solid mechanics, that were extended later to many other physics, like flows in narrow gaps, thermal or electromagnetic problems in laminates, among many others. However, in many cases, when addressing complex coupled physics the validity of hypotheses able to reduce models from 3D to 2D becomes doubtful and consequently in order to ensure accurate Results 3D discretizations seem compulsory. However the last imply too fine meshes when considering well-experienced mesh-based discretization procedures, where the mesh size is almost determined

\footnotetext{
* Corresponding author.

E-mail addresses: Giacomo.Quaranta@eleves.ec-nantes.fr (G. Quaranta), brice. bognet@ec-nantes.fr (B. Bognet), Ruben.Ibanez-Pinillo@eleves.ec-nantes.fr (R. Ibañez), Alain.Tramecon@esi-group.com (A. Tramecon), Eberhard.Haug@ esi-group.com (E. Haug), Francisco.CHINESTA@ensam.edu (F. Chinesta).
}

by the domain thickness and the material and/or solution details to be represented. In order to alleviate the associate computational complexity authors proposed few years ago computing the fully 3D solution employing an in-plane-out-of-plane separated representation whose computational complexity remains the one characteristic of 2D plate or shell simulations [5,6].

In many structural analysis and simulation of forming processes dynamical aspects cannot be neglected and then elastic models are replaced by their elastodynamics counterparts. It exists a vast literature on structural dynamics, covering different discretization techniques and time integration procedures $[29,17,27,30,26]$.

When considering an implicit analysis, solution at each time step needs some iterations to enforce equilibrium. On the contrary explicit schemes do not require iteration as the nodal accelerations are solved directly, and from which velocities and displacements are calculated by simple integration. At its turn displacements allow the calculation of strains and stresses. The main handicap of explicit simulations is that the time step must verify the stability condition, decreasing with the element size.

On the contrary implicit elastodynamic integrations become unconditionally stable, that is, there is not a limit in the time step to be considered in what concerns stability. Thus, implicit time steps are generally several orders of magnitude larger than the 
ones considered in explicit time integrations. However, implicit integration requires the solution of linear systems several times at each loading step when addressing nonlinear models. Explicit techniques do not require that matrix inversion and consequently address nonlinearities (contact or material nonlinearities) easily. In [18] a hybrid schema was proposed that considers the domain composed of two parts in which explicit and implicit time integrations apply.

When dynamics applies on degenerated domains, like plates or shells, and no acceptable simplifying hypotheses are available for reducing their complexity to $2 \mathrm{D}$, fully $3 \mathrm{D}$ solutions seem compulsory. This is for example the case when considering the progressive dynamic damage of composite laminates where a rich throughthe-thickness description could be extremely valuable, among many other scenarios in which a fully 3D formulation is retained.

In plane-out-of-plane separated representations, revisited in the next section, allows reducing the $3 \mathrm{D}$ solution to a sequence of 2D (in-plane) and 1D (along the thickness) problems, as proved when considering elastostatics in plate and shell domains $[5,6]$.

Even if the implementation of implicit integration schemes into a 3D in-plane-out-of-plane separated representation does not imply major difficulties, the use of explicit integration, preferable in many applications, e.g. crash simulations, becomes a tricky issue. In fact the mesh employed for discretizing the out-of-plane dimension (thickness) determines the limit time-step ensuring stability, and consequently it could become quickly unaffordable when refining the out-of-plane discretization.

In this paper we introduce a new hybrid explicit/implicit inplane-out-of-plane separated representation for dynamic problems defined in plate-like domains that computes efficiently 3D solution and where the stability constraints are exclusively determined by the coarser in-plane discretizations.

Next section revisits the main concepts related to the separated representations intensively considered in the present work. Then, Section 3 defines the elastodynamics problem and its in-plane-outof-plane separated representation. Section 4 addresses time integration within the separated representation framework, and proposes an efficient hybrid explicit/implicit formulation. Finally Section 5 validates the proposed methodology from some case studies.

\section{An overview on separated representations}

Separated representations, at the heart of the so-called Proper Generalized Decomposition - PGD - consists of expressing the unknown field depending on many coordinates (space, time, parameters, ...) as a finite sum decomposition in which each term involved in the sum consists at its turn in the product of a series of unknown functions each one depending on one coordinate. Thus, when addressing a transient model involving the unknown field $u(\mathbf{x}, t)$, its separated representation reads [20-22]

$u(\mathbf{x}, t) \approx \sum_{i=1}^{N} X_{i}(\mathbf{x}) \cdot T_{i}(t)$

where neither the time-dependent functions $T_{i}(t)$ nor the space functions $X_{i}(\mathbf{x})$ are "a priori" known. Both will be computed onthe-flight when solving the problem.

This rationale can be extended to the solution of any problem whose solution involves $d$ generic coordinates $u\left(x_{1}, \cdots, x_{d}\right)$ according to [1-3]

$u\left(x_{1}, x_{2}, \cdots, x_{d}\right) \approx \sum_{i=1}^{N} X_{i}^{1}\left(x_{1}\right) \cdot X_{i}^{2}\left(x_{1}\right) \cdots X_{i}^{d}\left(x_{d}\right)$,

where the set of coordinates can include a series of parameters $u\left(\mathbf{x}, t, p_{1}, \cdots, p_{\mathrm{p}}\right)$ according to [8] $u\left(\mathbf{x}, t, p_{1}, \cdots, p_{\mathrm{p}}\right) \approx \sum_{i=1}^{N} X_{i}(\mathbf{x}) \cdot T_{i}(t) \cdot \prod_{k=1}^{\mathrm{p}} P_{i}^{k}\left(p_{k}\right)$.

In the present paper we are mainly concerned by the space separation to address the solution of mechanical problems defined in degenerated domain. Next section revisits space separated representations.

Sometimes the spatial domain $\Omega$, assumed three-dimensional, can be fully or partially separated, and consequently it can be expressed as $\Omega=\Omega_{x} \times \Omega_{y} \times \Omega_{z}$ or $\Omega=\Omega_{x y} \times \Omega_{z}$ respectively. The first decomposition is related to hexahedral domains whereas the second one is related to plate, beams or extruded domains. We consider below both scenarii:

- The spatial domain $\Omega$ is partially separable. In this case the separated representation writes:

$$
u(\mathbf{x}, z) \approx \sum_{i=1}^{N} X_{i}(\mathbf{x}) \cdot Z_{i}(z)
$$

where $\mathbf{x}=(x, y) \in \Omega_{x y}$ and $z \in \Omega_{z}$. This decomposition implies:

(1) the solution in $\Omega_{x y}$ of two-dimensional BVP's to obtain functions $X_{i}$,

(2) the solution in $\Omega_{z}$ of one-dimensional BVP's to obtain functions $Z_{i}$.

The complexity of the PGD simulation scales with the twodimensional mesh used to solve the BVP's in $\Omega_{x y}$, regardless of the mesh used in the solution of the BVP defined in $\Omega_{z}$ for calculating $Z_{i}(z)$.

- The spatial domain $\Omega$ is fully separable. In this case the separated representation writes:

$$
u(x, y, z)=\sum_{i=1}^{N} X_{i}(x) \cdot Y_{i}(y) \cdot Z_{i}(z)
$$

implying:

(1) the solution in $\Omega_{X}$ of one-dimensional BVP's to obtain functions $X_{i}$,

(2) the solution in $\Omega_{y}$ of one-dimensional BVP's to obtain functions $Y_{i}$,

(3) the solution in $\Omega_{z}$ of one-dimensional BVP's to obtain functions $Z_{i}$.

The cost savings provided by the PGD are potentially phenomenal when the spatial domain is fully separable. Indeed, the complexity of the PGD simulation now scales with the one-dimensional meshes used to solve the BVP's in $\Omega_{x}, \Omega_{y}$ and $\Omega_{z}$.

Even when the domain is not fully separable, a fully separated representation could be considered by using appropriate geometrical mappings or by immersing the non-separable domain into a fully separable one. The interested reader can refer to [16,13].

In-plane-out-of-plane separated representations are particularly useful for addressing the solution of problems defined in plate [5], shell [6], beams [7] or extruded domains [23]. The same approach was extensively considered in structural plate and shell models in $[11,31-35,28]$. Space separated representations where enriched with discontinuous functions for representing cracks in [15], delamination in [24] and thermal contact resistances in [10]. Domain decomposition within the separated space representation was accomplished in [25]. The in-plane-out-of-plane decomposition was then extended to many other physics. Thermal models were considered in [10] and squeeze flows of Newtonian and non Newtonian fluids in laminates in $[12,14,19]$.

As soon as separated representations are considered the solution of a multidimensional problem reduces to the solution of a sequence of lower dimensional problems with the consequent 
computing time savings. The solution procedure has been extensively used, described and analyzed in our former works, many of them referred in the present work. The interested reader can refer to the primer [9] and the numerous references therein.

\section{Elastodynamics: problem definition}

We consider a physical domain $\Omega$ for which a linear elastic behavior is assumed, according to

$\boldsymbol{\sigma}=\mathbf{C}: \boldsymbol{\epsilon}$,

where $\mathbf{C}$ is the fourth order stiffness tensor, and the strain tensor $\boldsymbol{\epsilon}$ derives from the symmetric component of the gradient of displacements i.e. $\boldsymbol{\epsilon}=\nabla_{s} \mathbf{u}$, where $\nabla_{s}$ refers to the symmetric component.

From now on we consider Voigt notation, and for the sake of notational simplicity we consider the same notation, $\boldsymbol{\sigma}, \boldsymbol{\epsilon}$ and $\mathbf{C}$ for expressing the stress and strain vectors and the stiffness matrix respectively.

The dynamic problem, in absence of damping and external forces, with the displacement field $\mathbf{u}(\mathbf{x}, t)$ for $\mathbf{x} \in \Omega$ and $t \in I=[0, T]$, reads

$\rho \ddot{\mathbf{u}}(\mathbf{x}, t)=\nabla \cdot \boldsymbol{\sigma}$,

with $\rho$ the material density, $\dot{\mathbf{u}}$ and $\ddot{\mathbf{u}}$ the first and second time derivative of the displacement field respectively, i.e. the velocity and acceleration.

The domain boundary $\Gamma=\partial \Omega$ is partitioned in the so-called Dirichlet and Neumann regions, $\Gamma_{D}$ and $\Gamma_{N}$, where respectively displacements and tractions are enforced, with $\Gamma_{D} \cup \Gamma_{N}=\Gamma$ and $\Gamma_{D} \cap \Gamma_{N}=\varnothing$. Dynamic problems require specifying the initial displacement and velocity that without loss of generality in what follows are assumed null, i.e. $\dot{\mathbf{u}}(\mathbf{x}, t=0)=\mathbf{0}$ and $\mathbf{u}(\mathbf{x}, t=0)=\mathbf{0}$.

Assuming again the trial and test displacements belonging to appropriate functional spaces, and considering an elastic constitutive equation, the weak form associated with (7) reads

$\rho \int_{\Omega} \mathbf{u}^{*} \cdot \ddot{\mathbf{u}} d \mathbf{x}+\int_{\Omega} \boldsymbol{\epsilon}\left(\mathbf{u}^{*}\right) \cdot(\mathbf{C} \boldsymbol{\epsilon}(\mathbf{u})) d \mathbf{x}=\int_{\Gamma_{N}} \mathbf{u}^{*} \cdot \mathbf{F} d \mathbf{x}$,

where the applied traction depends on time, i.e. $\mathbf{F}=\mathbf{F}(t)$.

\subsection{In-plane-out-of-plane separated representation}

As discussed in the previous section, with $\Omega$ having one dimension (the one related to the thickness) much smaller than the others involving the in-plane coordinates, an in-plane-out-ofplane separated representation seems again the most appealing route for addressing 3D discretizations while keeping the computational complexity the one characteristic of 2D discretizations. The domain is expressed from $\Omega=\Omega_{x y} \times \Omega_{z}$.

Even if as also indicated space-time separated discretizations were considered many times in the past $[20,2]$, in the present work time derivatives are discretized using standard schemes.

By considering the notation $\mathbf{u}(x, y, z, t=k \Delta t)=\mathbf{u}^{k}(x, y, z)$, with $\Delta t$ the time step, the in-plane-out-of-plane separated representation of the displacement field at time $t_{k}=k \Delta t, \mathbf{u}^{k}(x, y, z)$, reads

$$
\begin{aligned}
\mathbf{u}^{k}(x, y, z) & =\left(\begin{array}{c}
u^{k}(x, y, z) \\
v^{k}(x, y, z) \\
w^{k}(x, y, z)
\end{array}\right) \approx \mathbf{u}_{N}^{k}(x, y, z) \\
& =\sum_{i=1}^{N}\left(\begin{array}{c}
u_{x y}^{i, k}(x, y) \cdot u_{z}^{i, k}(z) \\
v_{x y}^{i, k}(x, y) \cdot v_{z}^{i, k}(z) \\
w_{x y}^{i, k}(x, y) \cdot w_{z}^{i, k}(z)
\end{array}\right)=\sum_{i=1}^{N} \mathbf{U}_{x y}^{i, k}(x, y) \circ \mathbf{U}_{z}^{i, k}(z)
\end{aligned}
$$

where "o" refers to the Hadamard product, and with
$\mathbf{U}_{x y}^{i, k}(x, y)=\left(\begin{array}{c}u_{x y}^{i, k}(x, y) \\ v_{x y}^{i, k}(x, y) \\ w_{x y}^{i, k}(x, y)\end{array}\right)=\left(\begin{array}{c}u_{x y}^{i, k} \\ v_{x y}^{i, k} \\ w_{x y}^{i, k}\end{array}\right)$,

$\mathbf{U}_{z}^{i, k}(z)=\left(\begin{array}{c}u_{z}^{i, k}(z) \\ v_{z}^{i, k}(z) \\ w_{z}^{i, k}(z)\end{array}\right)=\left(\begin{array}{c}u_{z}^{i, k} \\ v_{z}^{i, k} \\ w_{z}^{i, k}\end{array}\right)$,

where for alleviating the notation the coordinate dependences will be omitted.

From all them we can obtain the separated vector form of the strain tensor at time $t_{k}, \boldsymbol{\epsilon}^{k} \equiv \boldsymbol{\epsilon}\left(\mathbf{u}^{k}\right)$ :

$$
\boldsymbol{\epsilon}\left(\mathbf{u}^{k}\right) \approx \sum_{i=1}^{N}\left(\begin{array}{c}
\frac{\partial u_{x y}^{i, k}}{\partial x} \cdot u_{z}^{i, k} \\
\frac{\partial v_{x y}^{i, k}}{\partial y} \cdot v_{z}^{i, k} \\
w_{x y}^{i, k} \cdot \frac{\partial w_{z}^{i, k}}{\partial z} \\
\frac{\partial u_{x y}^{i, k}}{\partial y} \cdot u_{z}^{i, k}+\frac{\partial v_{x y}^{i, k}}{\partial x} \cdot v_{z}^{i, k} \\
\frac{\partial w_{x y}^{i, k}}{\partial x} \cdot w_{z}^{i, k}+u_{x y}^{i, k} \cdot \frac{\partial u_{z}^{i, k}}{\partial z} \\
\frac{\partial w_{x y}^{i, k}}{\partial y} \cdot w_{z}^{i, k}+v_{x y}^{i, k} \cdot \frac{\partial v_{z}^{i, k}}{\partial z}
\end{array}\right) .
$$

The separated representation constructor proceeds by computing a term of the sum at each iteration. Assuming that the first $n-1$ modes (terms of the finite sum) of the solution were already computed, $\mathbf{u}_{n-1}^{k}(x, y, z)$ with $n \geqslant 1$, the solution enrichment reads:

$\mathbf{u}_{n}^{k}(x, y, z)=\mathbf{u}_{n-1}^{k}(x, y, z)+\mathbf{U}_{x y}^{n, k}(x, y) \circ \mathbf{U}_{z}^{n, k}(z)$

where both vectors $\mathbf{U}_{x y}^{n, k}$ and $\mathbf{U}_{z}^{n, k}$ are unknown at the present iteration defining a nonlinear problem. The test function $\mathbf{u}^{*}$ reads $\mathbf{u}^{*}=\mathbf{U}_{x y}^{*} \circ \mathbf{U}_{z}^{n, k}+\mathbf{U}_{x y}^{n, k} \circ \mathbf{U}_{z}^{*}$.

With both $\mathbf{U}_{x y}^{n, k}$ and $\mathbf{U}_{z}^{n, k}$ unknown the resulting problem becomes non-linear. We proceed by considering the simplest linearization strategy, an alternated directions fixed point algorithm widely considered and described in our former works.

When $\mathbf{U}_{z}^{n, k}$ is assumed known, we consider the test function $\mathbf{u}^{\star}$ given by $\mathbf{U}_{x y}^{\star} \circ \mathbf{U}_{z}^{n, k}$. By introducing the trial and test functions into the weak form and then integrating in $\Omega_{z}$ because all the functions depending on the thickness coordinate are known, we obtain a 2D weak formulation defined in $\Omega_{x y}$ whose discretization (by using a standard discretization strategy, e.g. finite elements) allows computing $\mathbf{U}_{x y}^{n, k}$.

Analogously, when $\mathbf{U}_{x y}^{n, k}$ is assumed known, the test function $\mathbf{u}^{\star}$ is given by $\mathbf{U}_{x y}^{n, k} \circ \mathbf{U}_{z}^{\star}$. By introducing the trial and test functions into the weak form and then integrating in $\Omega_{x y}$ because all the functions depending on the in-plane coordinates $(x, y)$ are at present known, we obtain a 1D weak formulation defined in $\Omega_{z}$ whose discretization (using any technique for solving standard ODE equations) allows computing $\mathbf{U}_{z}^{n, k}$.

Thus, the 3D computational cost is transformed into a sequence of 2D and 1D solutions, with the associated computing time savings [5].

\section{Time discretization}

Before introducing the hybrid strategy we consider at time $t_{k+1}$ the standard implicit and explicit formulations (two commun time integration schemas among other possibilities [4]), given respectively by

$$
\begin{aligned}
& \rho \int_{\Omega} \mathbf{u}^{*} \cdot \frac{\mathbf{u}^{k+1}-2 \mathbf{u}^{k}+\mathbf{u}^{k-1}}{\Delta t^{2}} d \mathbf{x}+\int_{\Omega} \boldsymbol{\epsilon}\left(\mathbf{u}^{*}\right) \cdot\left(\mathbf{C} \boldsymbol{\epsilon}\left(\frac{\mathbf{u}^{k+1}+\mathbf{u}^{k-1}}{2}\right)\right) d \mathbf{x} \\
& \quad=\int_{\Gamma_{N}} \mathbf{u}^{*} \cdot \frac{\mathbf{F}^{k+1}+\mathbf{F}^{k-1}}{2} d \mathbf{x}
\end{aligned}
$$


that as previously indicated is unconditionally stable, and the explicit one

$$
\begin{aligned}
& \rho \int_{\Omega} \mathbf{u}^{*} \cdot \frac{\mathbf{u}^{k+1}-2 \mathbf{u}^{k}+\mathbf{u}^{k-1}}{\Delta t^{2}} d \mathbf{x}+\int_{\Omega} \boldsymbol{\epsilon}\left(\mathbf{u}^{*}\right) \cdot\left(\mathbf{C} \boldsymbol{\epsilon}\left(\mathbf{u}^{k}\right)\right) d \mathbf{x} \\
& \quad=\int_{\Gamma_{N}} \mathbf{u}^{*} \cdot \mathbf{F}^{k} d \mathbf{x},
\end{aligned}
$$

that is conditionally stable, with the stability limit $\Delta t_{\max }$, defining the stability domain $\Delta t<\Delta t_{\max }$, given by

$\Delta t_{\max }=\frac{L}{c}$,

where $L$ is the characteristic length of the spatial discretization and the dilatational wave speed $c$ is given by

$c=\sqrt{\frac{E(1-v)}{(1+v)(1-2 v) \rho}}$.

As previously commented explicit strategies are employed in many commercial codes. However, when applied to discretize 3D problems defined in degenerated domains, like plates or shells, the extremely fine meshes considered along the thickness direction have an unfavorable impact on the time step that becomes extremely small to ensure stability. The in-plane-out-of-plane separated representation cannot scape to this important issue, being the mesh size along the out-of-plane coordinate (much finer that the one used in the plane) the one that determines the time step.

It is important emphasizing the main aim of the present work and the proposed methodology for performing it. First, it is important to note that we are interested in performing fully 3D simulations in degenerated geometries (e.g. plate domains) while considering explicit time integrations.

In this context the following remarks can be addressed:

- When using 2D discrete models (considering for example plate elements), the stability criterion related to explicit time integrations involves the size of the elements, but as the mesh is the one related to the middle plane, the critical time step remains reasonable in most of cases.

- However, as soon as 3D discretizations are considered, the characteristic size of the finite elements along the plate thickness becomes much smaller than the in-plane characteristic length, and then when considering explicit time integrations the time step needed for ensuring stability decreases with the throughof-thickness characteristic element length.

- Increasing the resolution in the thickness direction implies the increase of the number of elements involved in the discretization as well as the decrease of the time step for ensuring stability, both having unfavorable consequences on the computational cost.

- In our former works $[5,6]$ we proposed in the framework of elastostatics considering in-plane-out-of-plane separated representations that allowed reducing the computational complexity of solving a fully $3 \mathrm{D}$ problem to the one characteristic of 2D solutions.

- However, as just indicated, such a decomposition when combined with explicit time integrations fails, because again the stability is associated to the smallest discretization characteristic length, the one related to the through-of-thickness discretization.

- It is in that impasse that one is tempted of using, in the case of explicit time integration, the in-plane-out-of-plane separated representation (that reduces the computational complexity to the one characteristic of 2D models) combined with an hybrid time integration, explicit in the plane (conditionally stable but with the critical time-step scaling with the characteristic inplane discretization length) and implicit along the thickness (unconditionally stable), that allows reducing the computational complexity while keeping as stability constraint the one associated to the in-plane explicit time integration.
- Obviously fully implicit in-plane-out-of-plane decompositions are possible, where the implicit time integration ensures unconditional stability while the space separated representation reduces the computational complexity. Despite of its intrinsic interest it is not considered in the present paper, and in all cases, the associated solutions are the same as the ones obtained by using a fully 3D finite element discretization but reducing the solution computational complexity. As previously commented fully explicit integrations fail because the too stringent stability conditions induced by the too fine through-of-thickness discretization.

- Thus, in this paper we analyze the intermediate procedure, the one in which the fine through-of-thickness representation is alleviated thanks to the use of the in-plane-out-of-plane space separated representation and its associated implicit unconditionally stable time integration. Thus, the stability of the resulting discretization is expected being induced by the in-plane mesh in which an explicit time integration is retained. The present paper is intended analyzing this hybrid methodology, and proving that in the case of fully explicit separated representations (as in the case of fully explicit 3D finite elements) the stability is dictated by the smallest characteristic discretization length (the one along the domain thickness). On the contrary when considering the hybrid scheme described in the next section, we expect the stability being dictated by the characteristic in-plane discretization length (being the trough-of-thinness discretization implicit).

In summary, the main goal is enriching explicit 2D plate and shell formulations widely employed in industry and commercial codes, with a fine through-of-thickness description (3D) without affecting unfavorably the integration stability.

\subsection{Explicit-in-plane/implicit-out-of-plane hybrid scheme}

As just indicated, in order to circumvent the just referred stability issues, we propose an out-of-plane implicit discretization (unconditionally stable) while the in-plane discretization (implying coarser meshes) makes use of an explicit schema. Thus, the stability is prescribed by the in-plane size mesh, several order of magnitude higher than the one associated to the thickness.

For that purpose we propose considering at time $t_{k}$ the strain defined by

$$
\begin{aligned}
& \boldsymbol{\epsilon}^{h}\left(\mathbf{u}^{k}\right)=\left(\begin{array}{c}
u_{, x}^{k} \\
v_{y}^{k} \\
\frac{w_{z}^{k+1}+w_{z}^{k-1}}{2} \\
\frac{v_{z}^{k+1}+v_{, z}^{k-1}}{2}+w_{, y}^{k} \\
\frac{u_{z z}^{k+1}+u_{z}^{k-1}}{2}+w_{, x}^{k} \\
u_{, y}^{k}+v_{, x}^{k}
\end{array}\right) \\
& \approx\left(\begin{array}{c}
\sum_{i=1}^{N_{k}} u_{x y, x}^{i, k} \cdot u_{z}^{i, k} \\
\sum_{i=1}^{N_{k}} v_{x y, y}^{i, k} \cdot v_{z}^{i, k} \\
\frac{\sum_{i=1}^{N_{k+1}} w_{x y}^{i, k+1} \cdot w_{z, z}^{i, k+1}+\sum_{i=1}^{N_{k-1}} w_{x y}^{i, k-1} \cdot w_{z, z}^{i, k-1}}{2} \\
\frac{\sum_{i=1}^{N_{k+1}} u_{x y}^{i, k+1} \cdot v_{z, z}^{i, k+1}+\sum_{i=1}^{N_{k-1}} v_{x y}^{i, k-1} \cdot v_{z, z}^{i, k+1}+\sum_{i=1}^{N_{k-1}} u_{x y}^{i, k-1} \cdot u_{z, z}^{, i, k-1}}{2}+\sum_{i=1}^{N_{k}} w_{x y, y}^{i, k} \cdot w_{i=1}^{N_{k}} w_{x y, x}^{i, k} \cdot w_{z}^{i, k} \\
\sum_{i=1}^{N_{k}} u_{x y, y}^{i, k} \cdot u_{z}^{i, k}+\sum_{i=1}^{N_{k}} v_{x y, x}^{i, k} \cdot v_{z}^{i, k}
\end{array}\right)
\end{aligned}
$$


where for the sake of notational simplicity the derivatives of function $u_{\text {e }}$ with respect the coordinate $x$ is noted by $u_{\bullet} x$ (and similarly for the other functions involved in the displacement components with respect to any coordinate). Moreover, the superscript $\bullet^{h}$ refers to its hybrid nature and $N_{k+1}, N_{k}, N_{k-1}$ are the number of products involved in the separated representation of the displacement at times steps $k+1, k$ and $k-1$ respectively.

It can be noticed that the derivatives involving the out-of-plane (thickness) coordinate are treated using an implicit schema whereas an explicit one is retained for the in-plane derivatives. Thus, the hybrid schema is some place in between standard implicit and explicit techniques, taking profit of the advantages of both them.

When using the hybrid schema the weak form at time $t_{k+1}$, consists of finding $\mathbf{u}^{k+1}$, verifying

$\int_{\Omega} \rho \mathbf{u}^{*} \cdot \frac{\mathbf{u}^{k+1}-2 \mathbf{u}^{k}+\mathbf{u}^{k-1}}{\Delta t^{2}} d \mathbf{x}+\int_{\Omega} \boldsymbol{\epsilon}\left(\mathbf{u}^{*}\right) \cdot\left(\mathbf{C} \epsilon^{h}(\mathbf{u})\right) d \mathbf{x}=\int_{\Gamma_{N}} \mathbf{u}^{*} \cdot \mathbf{F}^{k} d \mathbf{x}$.

To construct the separated representation of the solution at time $t_{k+1}$ we consider the standard procedure, assuming that $n-1$ terms have been already computed and that at the present iteration looks for the term $n$, according to

$$
\mathbf{u}_{n-1}^{k+1}(x, y, z)=\left(\begin{array}{c}
u_{n-1}^{k+1}(x, y, z) \\
v_{n-1}^{k+1}(x, y, z) \\
w_{n-1}^{k+1}(x, y, z)
\end{array}\right)=\sum_{i=1}^{n-1} \mathbf{U}_{x y}^{i, k+1}(x, y) \circ \mathbf{U}_{z}^{i, k+1}(z),
$$

with

$\mathbf{u}_{n}^{k+1}(x, y, z)=\mathbf{u}_{n-1}^{k+1}(x, y, z)+\mathbf{P}^{k+1}(x, y) \circ \mathbf{T}^{k+1}(z)$.

where for the sake of notational simplicity the unknown fields $\mathbf{U}_{x y}^{n, k+1}$ and $\mathbf{U}_{z}^{n, k+1}$ are referred by $\mathbf{P}^{k+1}(x, y)$ and $\mathbf{T}^{k+1}(z)$, with components

$$
\mathbf{P}^{k+1}(x, y)=\left(\begin{array}{l}
p_{u}^{k+1}(x, y) \\
p_{v}^{k+1}(x, y) \\
p_{w}^{k+1}(x, y)
\end{array}\right)
$$

and

$$
\mathbf{T}^{k+1}(z)=\left(\begin{array}{c}
t_{u}^{k+1}(z) \\
t_{v}^{k+1}(z) \\
t_{w}^{k+1}(z)
\end{array}\right)
$$

The linearity allows writing

$\boldsymbol{\epsilon}_{n}^{h}(x, y, z)=\boldsymbol{\epsilon}_{n-1}^{h}(x, y, z)+\boldsymbol{\epsilon}_{P T}^{h}(x, y, z)$

where

$$
\begin{aligned}
& \epsilon_{n-1}^{h}(x, y, z)=\left(\begin{array}{c}
u_{, x}^{k} \\
v_{y}^{k} \\
\frac{w_{n-1, z}^{k+1}+w_{, z}^{k-1}}{2} \\
\frac{v_{n-1, z}^{k+1}+v_{z}^{k-1}}{2}+w_{, y}^{k} \\
\frac{u_{n-1, z}^{k+1}+u_{, z}^{k-1}}{2}+w_{, x}^{k} \\
u_{, y}^{k}+v_{, x}^{k}
\end{array}\right)
\end{aligned}
$$

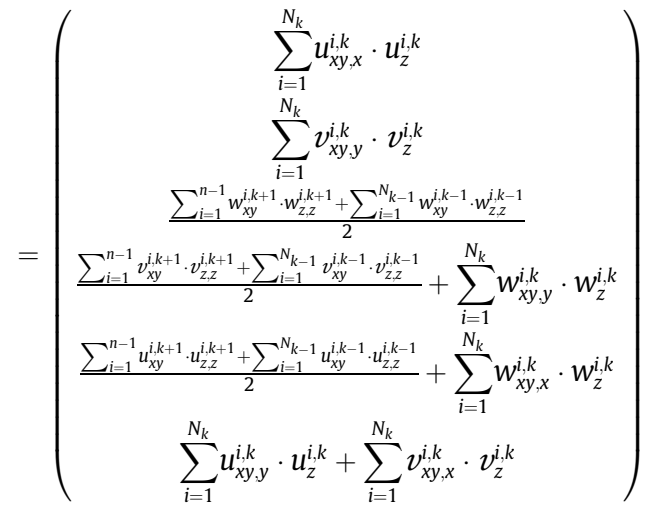

and

$\boldsymbol{\epsilon}_{P T}^{h}(x, y, z)=\left(\begin{array}{c}0 \\ 0 \\ \frac{p_{w}^{k+1} \cdot t_{w, z}^{k+1}}{2} \\ \frac{p_{v}^{k+1} \cdot t_{\nu, z}^{k+1}}{2} \\ \frac{p_{u}^{k+1} \cdot t_{u, z}^{k+1}}{2} \\ 0\end{array}\right)$

The test displacement reads

$$
\begin{aligned}
\mathbf{u}^{*}(x, y, z) & =\left(\begin{array}{l}
p_{u}^{*}(x, y) \cdot t_{u}^{k+1}(z)+p_{u}^{k+1}(x, y) \cdot t_{u}^{*}(z) \\
p_{v}^{*}(x, y) \cdot t_{v}^{k+1}(z)+p_{v}^{k+1}(x, y) \cdot t_{v}^{*}(z) \\
p_{w}^{*}(x, y) \cdot t_{w}^{k+1}(z)+p_{w}^{k+1}(x, y) \cdot t_{w}^{*}(z)
\end{array}\right) \\
& =\mathbf{P}^{*} \circ \mathbf{T}^{k+1}+\mathbf{P}^{k+1} \circ \mathbf{T}^{*}
\end{aligned}
$$

and the associated strain

$$
\boldsymbol{\epsilon}\left(\mathbf{u}^{*}(x, y, z)\right)=\left(\begin{array}{c}
p_{u, x}^{*} \cdot t_{u}^{k+1}+p_{u, x}^{k+1} \cdot t_{u}^{*} \\
p_{v, y}^{*} \cdot t_{v}^{k+1}+p_{v, y}^{k+1} \cdot t_{v}^{*} \\
p_{w}^{*} \cdot t_{w, z}^{k+1}+p_{w}^{k+1} \cdot t_{w, z}^{*} \\
p_{w, y}^{*} \cdot t_{w}^{k+1}+p_{w, y}^{k+1} \cdot t_{w}^{*}+p_{v}^{*} \cdot t_{v, z}^{k+1}+p_{v}^{k+1} \cdot t_{v, z}^{*} \\
p_{w, x}^{*} \cdot t_{w}^{k+1}+p_{w, x}^{k+1} \cdot t_{w}^{*}+p_{u}^{*} \cdot t_{u, z}^{k+1}+p_{u}^{k+1} \cdot t_{u, z}^{*} \\
p_{v, x}^{*} \cdot t_{v}^{k+1}+p_{v, x}^{k+1} \cdot t_{v}^{*}+p_{u, y}^{*} \cdot t_{u}^{k+1}+p_{u, y}^{k+1} \cdot t_{u}^{*}
\end{array}\right) .
$$

For the sake of simplicity, and without loss of generality, we assume that the applied traction $\mathbf{F}$ on $\Gamma_{N}$, can be expressed from the single term separated representation, i.e.

$\mathbf{F}^{k}(x, y, z)=\mathbf{F}_{x, y}^{k}(x, y) \circ \mathbf{F}_{z}^{k}(z)$.

Using the previous expressions, Eq. (19) reads

$$
\begin{aligned}
\int_{\Omega} \rho \mathbf{u}^{*} & \cdot \frac{\mathbf{P}^{k+1} \circ \mathbf{T}^{k+1}}{\Delta t^{2}} d \mathbf{x}+\int_{\Omega} \boldsymbol{\epsilon}\left(\mathbf{u}^{*}\right) \cdot\left(\mathbf{C} \epsilon_{P T}^{h}\right) d \mathbf{x} \\
= & -\int_{\Omega} \rho \mathbf{u}^{*} \cdot \frac{\mathbf{u}_{n-1}^{k+1}-2 \mathbf{u}^{k}+\mathbf{u}^{k-1}}{\Delta t^{2}} d \mathbf{x}-\int_{\Omega} \boldsymbol{\epsilon}\left(\mathbf{u}^{*}\right) \cdot\left(\mathbf{C} \epsilon_{n-1}^{h}\right) d \mathbf{x} \\
& +\int_{\Gamma_{N}} \mathbf{u}^{*} \cdot \mathbf{F}^{k} d \mathbf{x} .
\end{aligned}
$$

As both $\mathbf{P}^{k+1}$ and $\mathbf{T}^{k+1}$ are unknown, problem (29) becomes nonlinear and consequently requires an appropriate linearization strategy. As usual in our previous works an alternated directions fixed point strategy is considered that by assuming $\mathbf{T}^{k+1}$ known calculates $\mathbf{P}^{k+1}$ and from the last updates $\mathbf{T}^{k+1}$. The process continues until reaching convergence (the fixed point).

When assuming $\mathbf{T}^{k+1}$ known the test displacement reads

$\mathbf{u}^{*}(x, y, z)=\left(\begin{array}{l}p_{u}^{*}(x, y) \cdot t_{u}^{k+1}(z) \\ p_{v}^{*}(x, y) \cdot t_{v}^{k+1}(z) \\ p_{w}^{*}(x, y) \cdot t_{w}^{k+1}(z)\end{array}\right)=\mathbf{P}^{*} \circ \mathbf{T}^{k+1}$,

that introduced into the weak form (29) results in a 2D problem involving the in-plane coordinates that allows calculating $\mathbf{P}^{k+1}$. Now, assuming that last known, the test displacement becomes

$\mathbf{u}^{*}(x, y, z)=\left(\begin{array}{l}p_{u}^{k+1}(x, y) \cdot t_{u}^{*}(z) \\ p_{v}^{k+1}(x, y) \cdot t_{v}^{*}(z) \\ p_{w}^{k+1}(x, y) \cdot t_{w}^{*}(z)\end{array}\right)=\mathbf{P}^{k+1} \circ \mathbf{T}^{*}$,

that introduced at its turn into the weak form (29) results in a 1D problem involving the thickness, whose solution results in $\mathbf{T}^{k+1}$. As preciously indicated the iteration procedure continues until reaching the convergence characterized by 


$$
\frac{\int_{\Omega}\left(\mathbf{P}_{p}^{k+1} \circ \mathbf{T}_{p}^{k+1}-\mathbf{P}_{p-1}^{k+1} \circ \mathbf{T}_{p-1}^{k+1}\right)^{2} d \mathbf{x}}{\int_{\Omega}\left(\mathbf{P}_{p-1}^{k+1} \circ \mathbf{T}_{p-1}^{k+1}\right)^{2} d \mathbf{x}}<\epsilon,
$$

where $p$ refers to the fixed point iteration and $\epsilon$ is a small enough threshold value.

Similarly the enrichment procedure stops when the criterion

$$
\frac{\int_{\Omega}\left(\mathbf{U}_{x y}^{N_{k+1}, k+1} \circ \mathbf{U}_{z}^{N_{k+1}, k+1}\right)^{2} d \mathbf{x}}{\int_{\Omega}\left(\mathbf{U}_{x y}^{1, k+1} \circ \mathbf{U}_{z}^{1, k+1}\right)^{2} d \mathbf{x}}<\tilde{\epsilon},
$$

is fulfilled, with $\tilde{\epsilon}$ another small enough threshold value.

\section{Numerical validation}

\subsection{Dynamics of an homogeneous plate}

We consider the problem defined in the domain $\Omega$ depicted in Fig. 1, with $H_{x}=H_{y}=3 \mathrm{~m}$ and $H_{z}=0.1 \mathrm{~m}$. In the first case study, the material occupying $\Omega$ is assumed homogeneous.

Boundary conditions are given by: $\mathbf{u}=(0,0,0)$ on the face ADHE; $\mathbf{u}=$ (free, 0 , free) on the faces $\mathrm{ABFE}$ and $\mathrm{DCGH}$; $\mathbf{F}(\mathbf{t})=(0,0, A \sin (\omega t))$ on face BCGF, with $A=10^{8}, \omega=20 \pi$ as depicted in Fig. 2.

The material properties are defined in Table 1, where $E$ is the Young modulus, $v$ the Poisson coefficient and $\rho$ the material density.

Figs. 3 and 4 compare the stability of standard explicit Q8-3D finite elements (fully explicit separated representations with equivalent discretizations lead to the same results) and the hybrid scheme just proposed, for different values of the in-plane and outof-plane mesh sizes, $L_{x y}$ and $L_{z}$ respectively. As it can noticed computed results reflect the stability conditions given by Eqs. (16) and (17). It is important to note that when considering fully explicit schemes, the stability is found being prescribed by the mesh size related to the thickness direction, however, when considering the hybrid schema the stability becomes given by the in-plane characteristic mesh size, that being much larger that the one related to the thickness, integration becomes more efficient.

To validate the hybrid approach (only in what concerns accuracy and stability, because issues related to computing time savings were addressed in [5]), the computed solution is compared with both explicit and implicit 3D finite elements integration with a time step (in the explicit case) guaranteeing the integration stability. The simulation parameters are the ones introduced previously concerning the material properties, and the ones

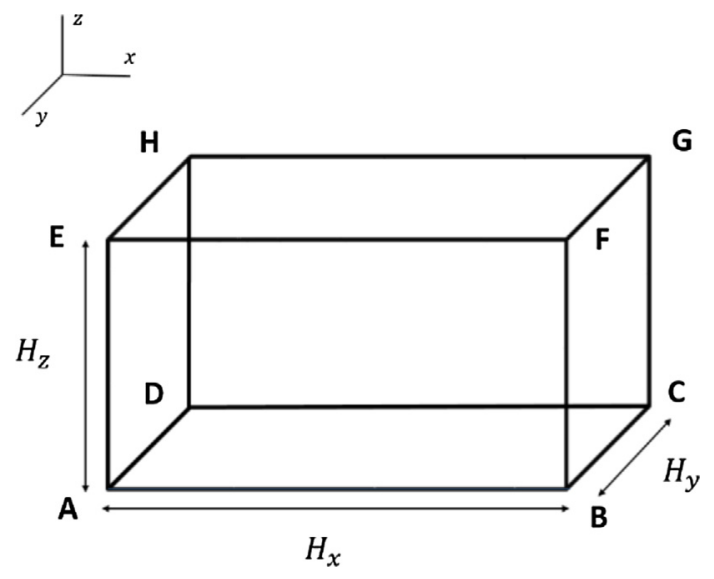

Fig. 1. Problem geometry.

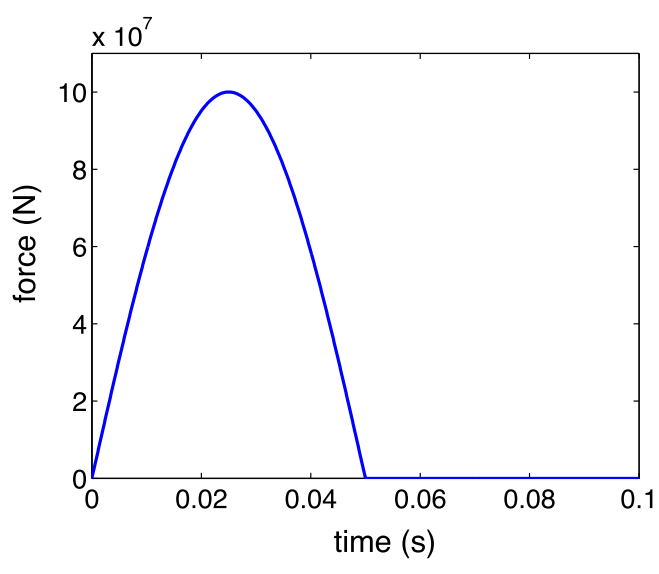

Fig. 2. Loading.

Table 1

Material properties.

\begin{tabular}{ll}
\hline$E\left(\mathrm{~N} / \mathrm{m}^{2}\right):$ & $2 \cdot 10^{11}$ \\
$v:$ & 0.25 \\
$\rho\left(\mathrm{kg} / \mathrm{m}^{3}\right):$ & 8000 \\
\hline
\end{tabular}

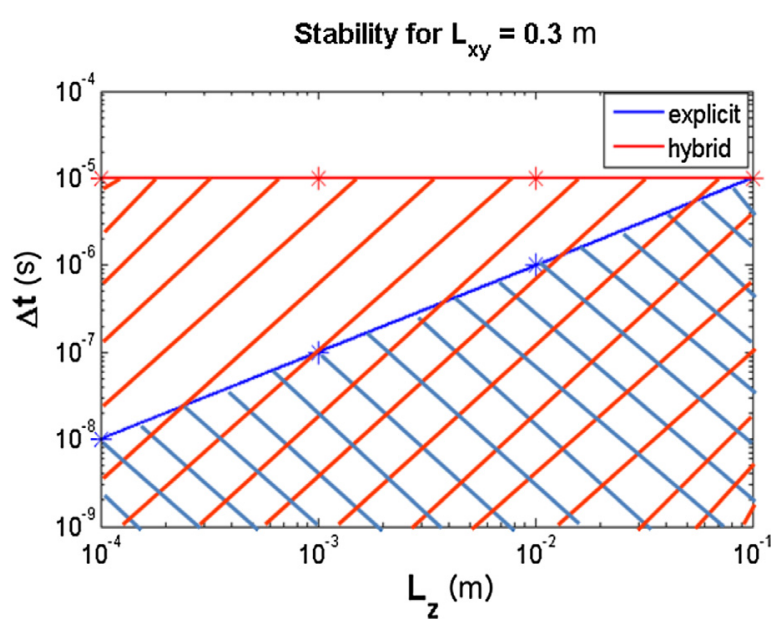

Fig. 3. Stability analysis for a given in-plane characteristic mesh size $L_{x y}$.

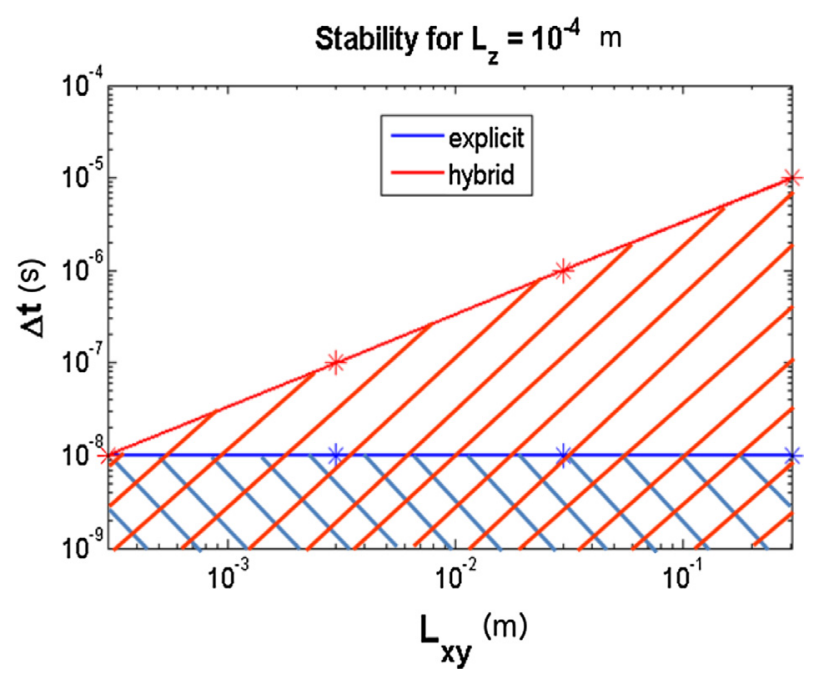

Fig. 4. Stability analysis for a given out-of-plane characteristic mesh size $L_{z}$. 
Table 2

Simulation parameters.

\begin{tabular}{ll}
\hline$H_{x}:$ & $3 \mathrm{~m}$ \\
$H_{y}:$ & $3 \mathrm{~m}$ \\
$H_{z}:$ & $0.1 \mathrm{~m}$ \\
$N_{x}:$ & 10 \\
$N_{y}:$ & 2 \\
$N_{z}:$ & 10 \\
$\Delta t:$ & $10^{-6} \mathrm{~s}$ \\
\hline
\end{tabular}

concerning the remaining simulation parameters are indicated in Table 2, where $N_{x}, N_{y}$ and $N_{z}$ refer to the number of elements involved in the discretization of directions $x, y$ and $z$.

Fig. 5 depicts the time evolution of the vertical displacement $w$ at the central point on segment FG when using different integration schemes. The solution obtained by using the hybrid strategy agrees in minute with the one obtained by using the finite element method and considered as reference for comparison purposes.

\subsection{Considering richer out-of-plane approximations}

In order to check the ability of the proposed technique for addressing richer out-of-plane representations, we consider that the domain depicted in Fig. 1 consists now in a laminated composed of 8 anisotropic plies $[0,45,-45,90]_{S}$. The applied force now writes again $\mathbf{F}(\mathbf{t})=(0,0, A \sin (\omega t))$ and applies on the face BCGF, with $A=10^{8}$ but now with $\omega=200 \pi$, as depicted in Fig. 6 .

The mechanical properties of the $0^{\circ}$-ply are given in Table 3, where $E$ is the Young modulus, $v$ the Poisson coefficient, $G$ the shear modulus and $\rho$ the density. The subscripts indicate respectively the proprieties along the longitudinal direction of the fibers (1), the in-plane transverse direction (2) and the out-of-plane direction (3).

In the present case the elastic constitutive equation becomes orthotropic and using again Voigt notation it reads

$$
\left[\begin{array}{c}
\varepsilon_{x x} \\
\varepsilon_{y y} \\
\varepsilon_{z z} \\
\gamma_{y z} \\
\gamma_{x z} \\
\gamma_{x y}
\end{array}\right]=\left[\begin{array}{cccccc}
\frac{1}{E_{1}} & -\frac{v_{12}}{E_{1}} & -\frac{v_{13}}{E_{1}} & 0 & 0 & 0 \\
-\frac{v_{12}}{E_{1}} & \frac{1}{E_{2}} & -\frac{v_{23}}{E_{2}} & 0 & 0 & 0 \\
-\frac{v_{13}}{E_{1}} & -\frac{v_{23}}{E_{2}} & \frac{1}{E_{3}} & 0 & 0 & 0 \\
0 & 0 & 0 & \frac{1}{G_{23}} & 0 & 0 \\
0 & 0 & 0 & 0 & \frac{1}{G_{13}} & 0 \\
0 & 0 & 0 & 0 & 0 & \frac{1}{G_{12}}
\end{array}\right]\left[\begin{array}{c}
\sigma_{x x} \\
\sigma_{y y} \\
\sigma_{z z} \\
\sigma_{y z} \\
\sigma_{x z} \\
\sigma_{x y}
\end{array}\right] .
$$

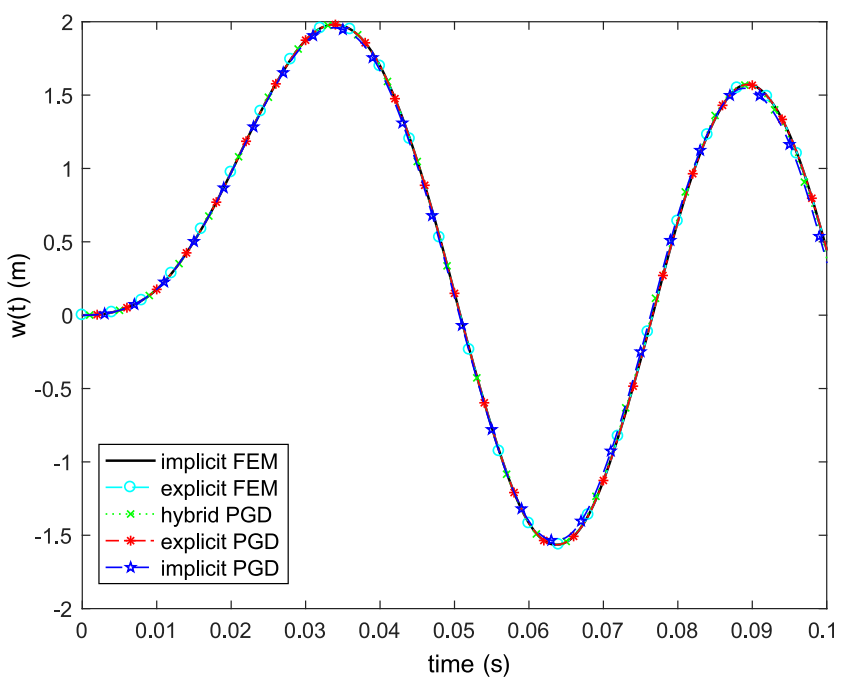

Fig. 5. Vertical displacement at the central point of segment FG.
We compared the solution obtained using the hybrid strategy with the one obtained using implicit finite elements. An explicit finite element solution was not envisaged because the too small time step induced by the extremely fine through-the-thickness mesh. The simulation parameters are reported in Tables 3 and 4.

Fig. 7 compares the time evolution of the vertical displacement at the middle of segment FG. It can be noticed again that the hybrid strategy provides an excellent solution compared with the implicit finite element considered as reference.

Finally Fig. 8 compares the time evolution of the stress component $\sigma_{z z}$ at the same location calculated (middle of segment FG) using the finite element method and the implicit/explicit hybrid in-plane-out-of-plane decomposition. Again both solutions match perfectly.

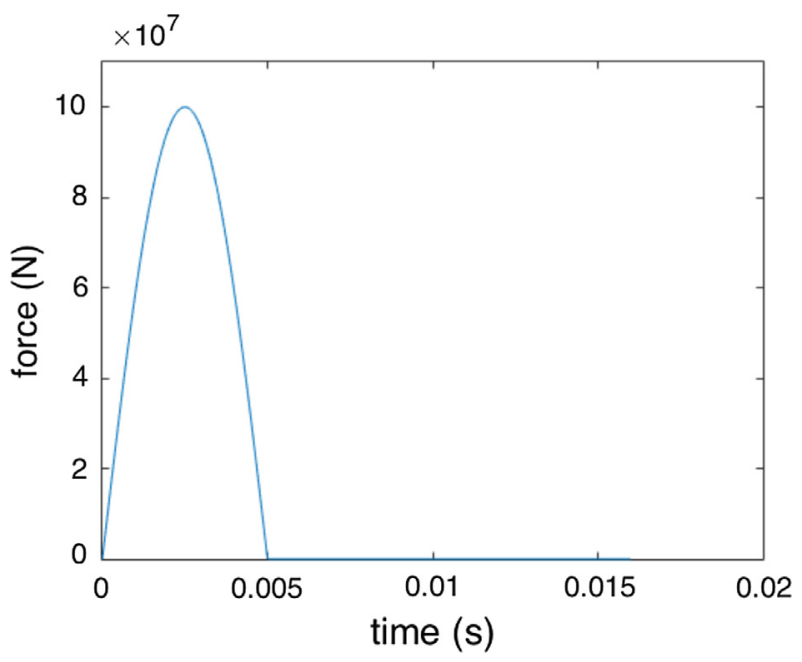

Fig. 6. Loading applying in the composite laminate.

Table 3

Mechanical properties of the $0^{\circ}$-ply.

$\begin{array}{ll}E_{1}\left(\mathrm{~N} / \mathrm{mm}^{2}\right): & 120 \cdot 10^{3} \\ E_{2}\left(\mathrm{~N} / \mathrm{mm}^{2}\right): & 8.9 \cdot 10^{3} \\ E_{3}\left(\mathrm{~N} / \mathrm{mm}^{2}\right): & 8.9 \cdot 10^{3} \\ v_{12}: & 0.35 \\ v_{13}: & 0.35 \\ v_{23}: & 0.32 \\ v_{21}: & v_{12} \\ v_{31}: & v_{13} \\ v_{32}: & v_{23} \\ G_{12}\left(\mathrm{~N} / \mathrm{mm}^{2}\right): & 4.5 \cdot 10^{3} \\ G_{13}\left(\mathrm{~N} / \mathrm{mm}^{2}\right): & 4.5 \cdot 10^{3} \\ G_{23}\left(\mathrm{~N} / \mathrm{mm}^{2}\right): & 5.3 \cdot 10^{3} \\ \rho\left(\mathrm{kg} / \mathrm{m}^{3}\right): & 1750\end{array}$

Table 4

Simulation parameters.

\begin{tabular}{ll}
\hline$H_{x}:$ & $250 \mathrm{~mm}$ \\
$H_{y}:$ & $100 \mathrm{~mm}$ \\
$H_{z}:$ & $4 \mathrm{~mm}$ \\
$N_{x}:$ & 10 \\
$N_{y}:$ & 2 \\
$N_{z}:$ & $48(6$ elements per ply) \\
$\Delta t:$ & $10^{-6} \mathrm{~s}$ \\
\hline
\end{tabular}




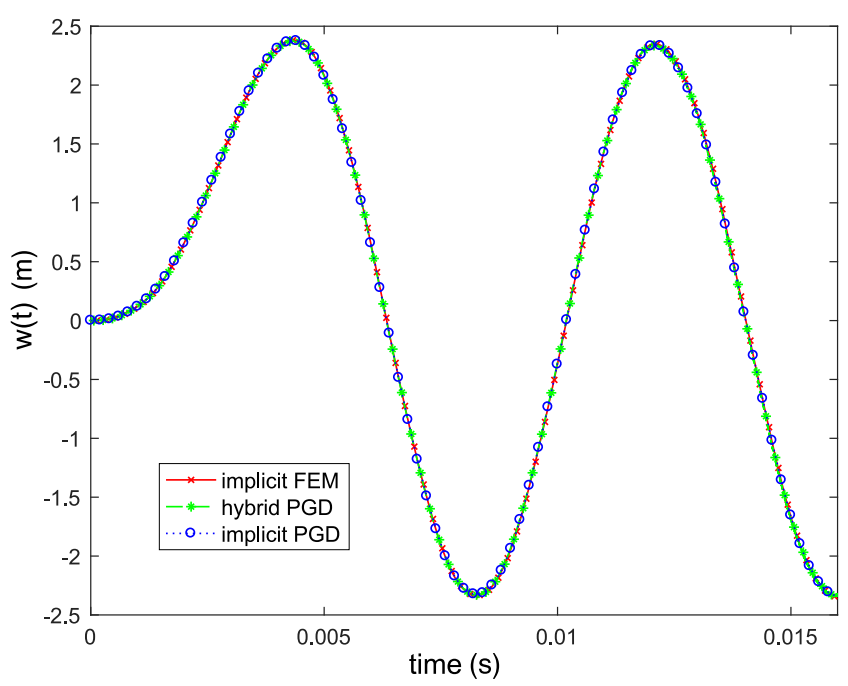

Fig. 7. Vertical displacement at the middle of segment FG.

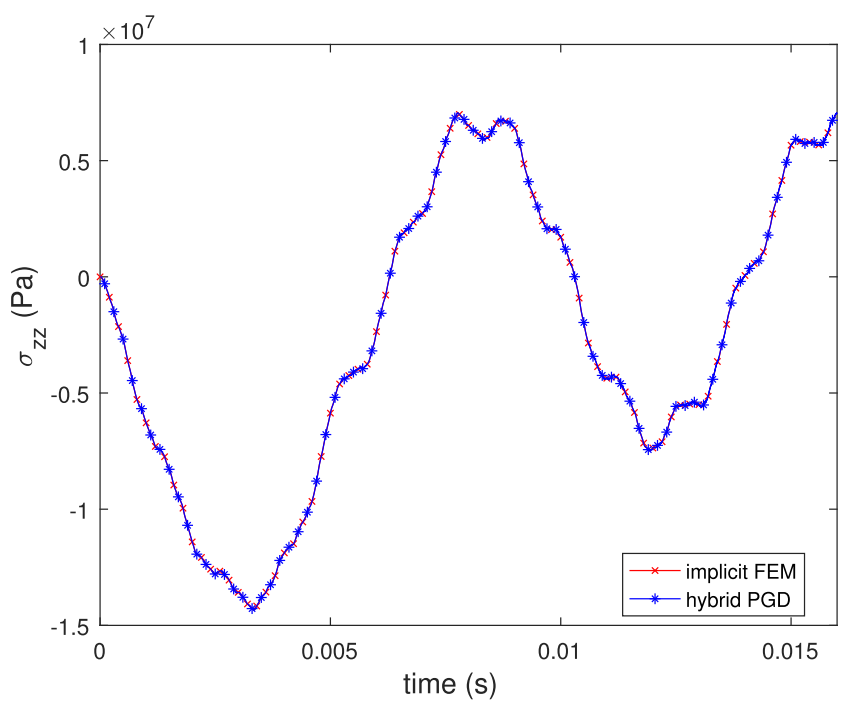

Fig. 8. Stress component $\sigma_{z z}(t)$ at the central point of segment FG. Implicit and hybrid-based solutions are almost superimposed.

\section{Analysis of computational performances}

In order to investigate the performances of the proposed technique we perform in this section different analyses. Before, we would like advertising on two facts. First, all computing times are referred to a Matlab implementation on a standard laptop. Thus, computed results allow comparing approaches but not to conclude on absolute performances. Second, for the sake of generality the problem linearity is not taken into account in the sense that at each time step a linear system is solved without taking advantage of the numerous computational profits that linearity offers in the finite element framework. This conservative approach allows extending the main conclusions to the nonlinear case.

We consider again the problem defined in the domain $\Omega$ depicted in Fig. 1, with $H_{x}=H_{y}=3 \mathrm{~m}, H_{z}=0.1 \mathrm{~m}$ and with the material properties defined in Table 1 , considering the same boundary conditions than in Section 5.1 and the same loading, the last illustrated in Fig. 2. In the analyses here addressed, the PGD constructor stoping criterion is set to $\epsilon=\tilde{\epsilon}=10^{-6}$.
First, we compare the hybrid PGD method with its fully implicit counterpart. The three different meshes defined in Table 5 are considered, where again $N_{x}, N_{y}$ and $N_{z}$ refer to the number of elements involved in the discretization of directions $x, y$ and $z$ respectively. For each mesh we compare the computing time employed by both the hybrid and the fully implicit PGD discretizations to solve the problem in the time interval $[0,400 \Delta t]$, with the time-step $\Delta t=\frac{10^{-5}}{3} \mathrm{~s}$ for all the simulations.

Results presented in Fig. 9 prove that, as expected, when using the same time-step the hybrid method proceeds faster than the implicit one. Later, in order to take advantage of the superior stability of fully implicit discretizations, time-steps will be selected differently for ensuring an equivalent accuracy, in order to compare computing costs in a more appropriate manner.

Now, we perform a comparison between the three PGD formulations (explicit, hybrid and implicit) in the time interval $[0,400 \Delta t]$, with $\Delta t=10^{-7} \mathrm{~s}$ to ensure the stability of the explicit time integration. Results for the three meshes in Table 6 are presented in Fig. 10. As expected the computational cost of the hybrid formulation is in between the one of explicit and implicit time integrations.

We have already proved in Figs. 3 and 4 that the stability domain of the hybrid formulation does not depend on the mesh size associated with the thickness direction, so that the hybrid simulation proceeds faster than the one performed using a fully explicit formulation by using a larger time-step in the hybrid integration.

Table 5

Meshes considered in the analysis of computational performances depicted in Fig. 9.

\begin{tabular}{cccc}
\hline & Mesh 1 & Mesh 2 & Mesh 3 \\
\hline$N_{x}:$ & 10 & 20 & 30 \\
$N_{y}:$ & 10 & 20 & 30 \\
$N_{z}:$ & 100 & 100 & 100 \\
\hline
\end{tabular}

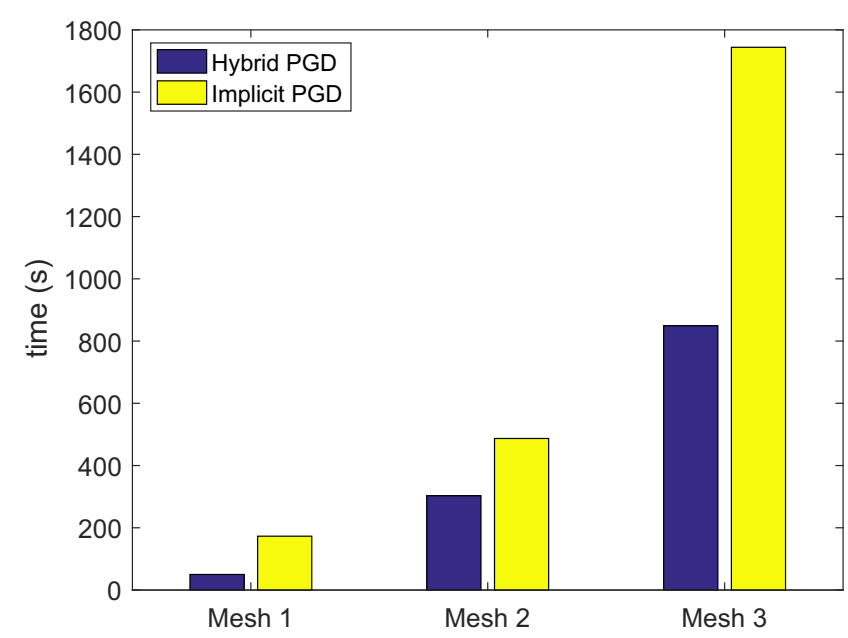

Fig. 9. Hybrid versus implicit PGD formulations.

Table 6

Meshes considered in the analysis of computational performances depicted in Fig. 10.

\begin{tabular}{cccc}
\hline & Mesh 4 & Mesh 5 & Mesh 6 \\
\hline$N_{x}:$ & 10 & 20 & 30 \\
$N_{y}:$ & 10 & 20 & 30 \\
$N_{z}:$ & 50 & 50 & 50 \\
\hline
\end{tabular}




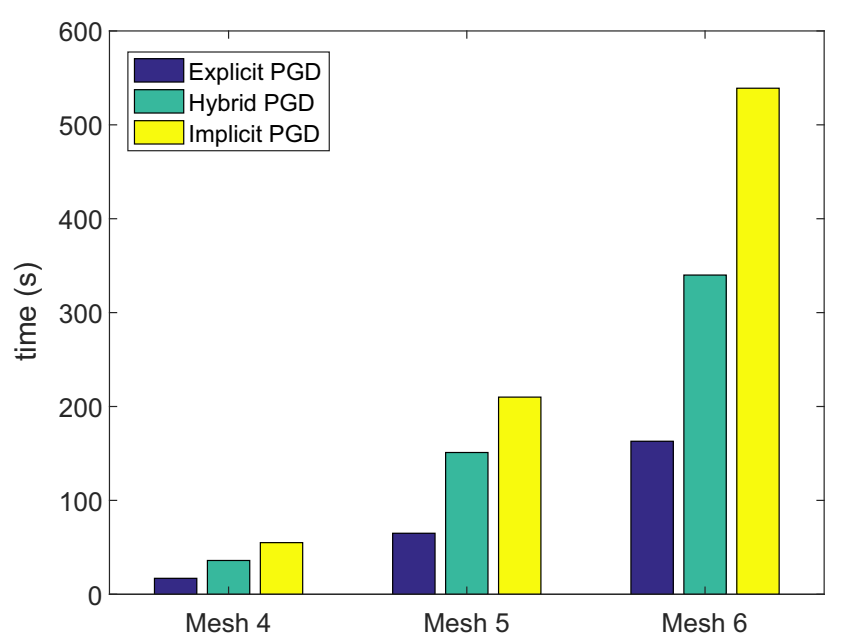

Fig. 10. Comparing explicit, hybrid and implicit PGD formulations.

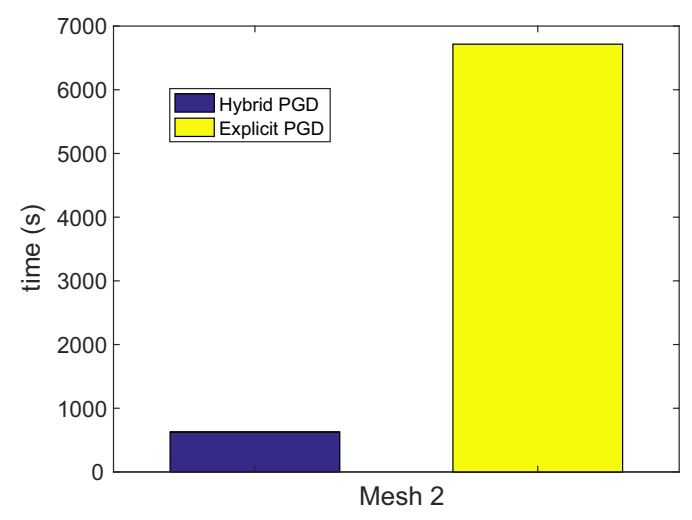

Fig. 11. Hybrid versus explicit PGD formulations.

In order to prove it, we perform a simulation using "Mesh 2" in Table 5 , in the time interval $[0,4] \mathrm{ms}$. We use as time steps for the explicit and the hybrid methods respectively $\Delta t_{e x}=10^{-7} \mathrm{~s}$ and $\Delta t_{h y}=10^{-5} \mathrm{~s}$, ensuring the stability of both schemes. Results shown in Fig. 11 reveal as expected that the higher time-step considered in the hybrid integration induces significant computing time savings.

The last analysis aims at taking advantage of the superior stability performances of the implicit formulation, that a priori can use larger time-steps that the ones of explicit and hybrid formulations that are only conditionally stables. However, here not only stability issues are addressed but also the accuracy of the computed solutions.

Thus, in the present numerical analysis we consider the mesh defined Table 7 and the time interval $[0, T]$ with $T=6 \mathrm{~ms}$, and consider as reference solution the one computed using an explicit FEM scheme with a very fine time-step $\Delta t=10^{-7} \mathrm{~s}$, ensuring both stability and accuracy, both performances having been checked. Fig. 12 compares the computational cost related to FEM and PGD explicit time integrations. As expected the separated representation involved in the PGD formulation allows better performances.

Then the problem is solved using first the hybrid scheme with a time-step $\Delta t=2 \cdot 10^{-5} \mathrm{~s}$ that ensures its stability and implicit (PGD and FEM) time integrations using higher time-steps. For each solution we consider the computational cost as well as the error $E$ with respect to the reference solution, computed from
Table 7

Mesh used in the results described in Fig. 12 and Table 8.

\begin{tabular}{cc}
\hline & Mesh 7 \\
\hline$N_{x}:$ & 10 \\
$N_{y}:$ & 10 \\
$N_{z}:$ & 90 \\
\hline
\end{tabular}

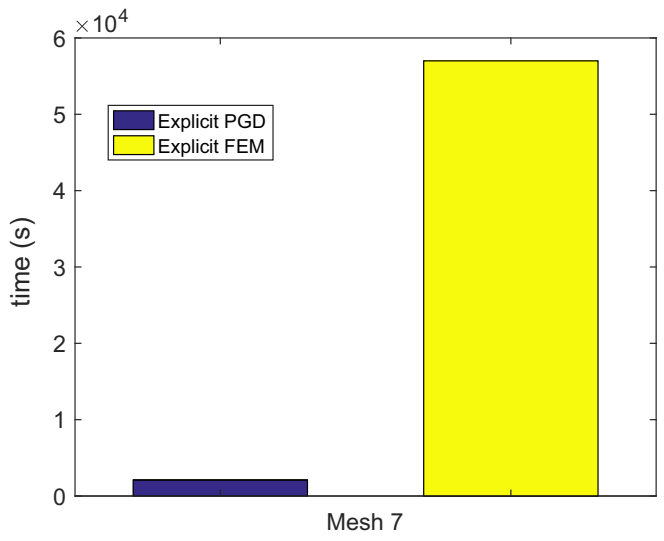

Fig. 12. Explicit PGD versus explicit FEM.

Table 8

Hybrid and implicit PGD integrations versus a standard implicit finite element formulation (using different time-steps).

\begin{tabular}{llcc}
\hline & & Time & Error \\
\hline \multirow{2}{*}{$\Delta t=2 \cdot 10^{-5} \mathrm{~s}$} & Hybrid PGD: & $65 \mathrm{~s}$ & $8.3 \cdot 10^{-3}$ \\
& Implicit PGD: & $203 \mathrm{~s}$ & $8.4 \cdot 10^{-3}$ \\
& Implicit FEM: & $746 \mathrm{~s}$ & $8.4 \cdot 10^{-3}$ \\
$\Delta t=4 \cdot 10^{-5} \mathrm{~s}$ & Implicit PGD: & $185 \mathrm{~s}$ & $1.66 \cdot 10^{-2}$ \\
& Implicit FEM: & $363 \mathrm{~s}$ & $1.66 \cdot 10^{-2}$ \\
$\Delta t=6 \cdot 10^{-5} \mathrm{~s}$ & Implicit PGD: & $145 \mathrm{~s}$ & $2.47 \cdot 10^{-2}$ \\
& Implicit FEM: & $236 \mathrm{~s}$ & $2.47 \cdot 10^{-2}$ \\
$\Delta t=8 \cdot 10^{-5} \mathrm{~s}$ & Implicit PGD: & $88 \mathrm{~s}$ & $3.27 \cdot 10^{-2}$ \\
& Implicit FEM: & $174 \mathrm{~s}$ & $3.27 \cdot 10^{-2}$ \\
$\Delta t=10^{-4} \mathrm{~s}$ & Implicit PGD: & $63 \mathrm{~s}$ & $4.05 \cdot 10^{-2}$ \\
& Implicit FEM: & $146 \mathrm{~s}$ & $4.05 \cdot 10^{-2}$ \\
\hline
\end{tabular}

$E=\frac{\left(\int_{0}^{T} \int_{\Omega}\left(\mathbf{u}-\mathbf{u}_{r e f}\right)^{2} d \mathbf{x} d t\right)^{\frac{1}{2}}}{\left(\int_{0}^{T} \int_{\Omega} \mathbf{u}_{r e f}^{2} d \mathbf{x} d t\right)^{\frac{1}{2}}}$.

Table 8 compares the different solutions, proving that: (i) implicit PGD and FEM integrations lead to almost the same solutions, being the ones related to PGD less computationally expensive; and (ii) implicit simulations related to the same computational cost than hybrid simulation produce larger errors, for the analyzed case.

\section{Conclusion}

This paper proposes a new time discretization scheme for solving 3D dynamical problems defined in degenerated domains, that is, domains in which one of its characteristic dimensions is much smaller that the other ones, as it is the case when considering plates or shells. 
A first complexity reduction is attained by considering an in-plane-out-of-plane separated representation that allows reducing the original 3D complexity to the one characteristic of 2D plate or shells models, even if the computed solution is fully 3D and any hypothesis is introduced. Such separated representation allows the use of extremely fine descriptions along the thickness direction.

However, such decomposition when combined with explicit time integrations has a major handicap, the too small size of the discretization involved in the thickness direction implies an extremely small time step for ensuring stability. In this paper we circumvent such a drawback by using an implicit (unconditionally stable) through-the-thickness discretization whereas a standard explicit scheme is considered for treating the in-plane operators. Thus, the stability is dictated by the in-plane mesh size, much coarser than the one employed in the thickness direction. It is important to note, that even if a part of the whole scheme remains implicit, it only affects one dimension and then its solution is extremely fast and cheap.

The inclusion of progressive damage models combined with dynamical effects constitutes a work in progress, where the separated representations seems an appealing option to better represent damage effects along the laminate thickness, and where explicit time integrations are usually employed in industrial applications.

\section{Acknowledgment}

Authors thanks ESI Group through its chair at Centrale Nantes, and in particular Drs. E. Abisset-Chavanne and J.V. Aguado, both involved in the chair and that contributed partially to the works and results here reported. They also thanks Prof. A. Huerta from UPC, Barcelona (Spain), for the valuable discussions. The research leading to these results has received funding from the People Programme (Marie Curie Actions) of the European Union's Seventh Framework Programme MSC - ITN - ETN under REA grant agreement [675919].

\section{References}

[1] Ammar A, Mokdad B, Chinesta F, Keunings R. A new family of solvers for some classes of multidimensional partial differential equations encountered in kinetic theory modeling of complex fluids. J Non-Newton Fluid Mech 2006; 139:153-76.

[2] Ammar A, Mokdad B, Chinesta F, Keunings R. A new family of solvers for some classes of multidimensional partial differential equations encountered in kinetic theory modeling of complex fluids. Part II: Transient simulation using space-time separated representation. J Non-Newton Fluid Mech 2007;144:98-121.

[3] Ammar A, Chinesta F, Joyot P. The nanometric and micrometric scales of the structure and mechanics of materials revisited: an introduction to the challenges of fully deterministic numerical descriptions. Int J Multiscale Comput Eng 2008;6/3:191-213.

[4] Bathe KJ. Finite element procedures. Prentice Hall, Pearson Education, Inc.; 2006.

[5] Bognet B, Leygue A, Chinesta F, Poitou A, Bordeu F. Advanced simulation of models defined in plate geometries: 3D solutions with 2D computational complexity. Comput Methods Appl Mech Eng 2012;201:1-12.

[6] Bognet B, Leygue A, Chinesta F. Separated representations of 3D elastic solutions in shell geometries. Adv Modell Simul Eng Sci 2014;1:4. https://doi. org/10.1186/2213-7467-1-4.

[7] Bordeu F, Ghnatios Ch, Boulze D, Carles B, Sireude D, Leygue A, et al. Parametric 3D elastic solutions of beams involved in frame structures. Adv Aircraft Spacecraft Sci 2015;2/3:233-48.
[8] Chinesta F, Leygue A, Bordeu F, Aguado JV, Cueto E, Gonzalez D, et al Parametric PGD based computational vademecum for efficient design, optimization and control. Arch Comput Methods Eng 2013;20/1:31-59.

[9] Chinesta F, Leygue A, Keunings R. The proper generalized decomposition for advanced numerical simulations. A primer. Springer Briefs in Applied Science and Technology, Springer; 2014.

[10] Chinesta F, Leygue A, Bognet B, Ghnatios Ch, Poulhaon F, Bordeu F, et al. First steps towards an advanced simulation of composites manufacturing by automated tape placement. Int J Mater Form 2014:7/1:81-92.

[11] Gallimard L, Vidal P, Polit O. Coupling finite element and reliability analysis through proper generalized decomposition model reduction. Int J Numer Meth Eng 2013;95/13:1079-93.

[12] Ghnatios Ch, Chinesta F, Binetruy Ch. The squeeze flow of composite laminates. Int J Mater Form 2015;8:73-83.

[13] Ghnatios Ch, Xu G, Visonneau M, Leygue A, Chinesta F, Cimetiere A. On the space separated representation when addressing the solution of PDE in complex domains. Discr Contin Dyn 2016;9/2:475-500.

[14] Ghnatios Ch, Abisset-Chavanne E, Binetruy Ch, Chinesta F, Advani S. 3D modeling of squeeze flow of multiaxial laminates. J Non-Newton Fluid Mech 2016;234:188-200.

[15] Giner E, Bognet B, Rodenas JJ, Leygue A, Fuenmayor J, Chinesta F. The Proper Generalized Decomposition (PGD) as a numerical procedure to solve 3D cracked plates in linear elastic fracture mechanics. Int J Solid Struct 2013;50 10:1710-20.

[16] Gonzalez D, Ammar A, Chinesta F, Cueto E. Recent advances in the use of separated representations. Int J Numer Meth Eng 2010;81/5:637-59.

[17] Gravouil A, Combescure A. Multitime-step explicit-implicit method for nonlinear structural dynamics. Int J Numer Meth Eng 2001;50:199-225.

[18] Hughes TJR, Liu WK. Implicit-explicit finite elements in transient analysis: stability theory. J Appl Mech 1978;45/2:371-4.

[19] Ibanez R, Abisset-Chavanne E, Chinesta F, Huerta A. Simulating squeeze flows in multiaxial laminates: towards fully 3D mixed formulations. Int J Mater Form. https://doi.org/10.1007/s12289-016-1309-4.

[20] Ladeveze P. On a family of algorithms for structural mechanics. C R Acad Sci Paris 1985;300/2:41-4 [in French].

[21] Ladeveze P. The large time increment method for the analyze of structures with nonlinear constitutive relation described by internal variables. C R Acad Sci Paris 1989;309:1095-9.

[22] Ladeveze P. Mécanique non linéaire des structures. Paris: Hermès; 1996.

[23] Leygue A, Chinesta F, Beringhier M, Nguyen TL, Grandidier JC, Pasavento F, et al. Towards a framework for non-linear thermal models in shell domains. Int J Numer Meth Heat Fluid Flow 2013;23/1:55-73.

[24] Metoui S, Pruliere E, Ammar A, Dau F, Iordanoff I. The proper generalized decomposition for the simulation of delamination using cohesive zone model Int J Numer Meth Eng 2014;99/13:1000-22.

[25] Nazeer M, Bordeu F, Leygue A, Chinesta F. Arlequin based PGD domain decomposition. Comput Mech 2014;54/5:1175-90.

[26] Pinho ST, Iannucci L, Robinson P. Formulation and implementation of decohesion elements in an explicit finite element code. Compos Part A: Appl Sci Manuf 2006;37/5:778-89.

[27] Prior AM. Applications of implicit and explicit finite element techniques to metal forming. J Mater Process Technol 1994;45:649-56.

[28] Pruliere E. 3D simulation of laminated shell structures using the Proper Generalized Decomposition. Compos Struct 2014;117:373-81.

[29] Sun JS, Lee KH, Lee HP. Comparison of implicit and explicit finite element methods for dynamic problems. J Mater Process Technol 2000;105:110-8.

[30] Taylor L, Cao J, Karafillis AP, Boyce MC. Numerical simulations of sheet-metal forming. J Mater Process Tech 1995;50:168-79.

[31] Vidal P, Gallimard L, Polit O. Composite beam finite element based on the Proper Generalized Decomposition. Comput Struct 2012;102:76-86.

[32] Vidal P, Gallimard L, Polit O. Proper Generalized Decomposition and layer-wise approach for the modeling of composite plate structures. Int J Solids Struct 2013;50/14-15:2239-50.

[33] Vidal P, Gallimard L, Polit O. Explicit solutions for the modeling of laminated composite plates with arbitrary stacking sequences. Compos Part B - Eng 2014;60:697-706

[34] Vidal P, Gallimard L, Polit O. Shell finite element based on the Proper Generalized Decomposition for the modeling of cylindrical composite structures. Comput Struct 2014;132:1-11.

[35] Vidal P, Gallimard L, Polit O. Assessment of variable separation for finite element modeling of free edge effect for composite plates. Compos Struct 2015;123:19-29. 